\title{
Le sourire
}

Un sourire ne coûte rien et produit beaucoup,

Il enrichit celui qui le reçoit sans appauvrir celui qui le donne,

Il ne dure qu'un instant, mais son souvenir est parfois éternel,

Personne n'est assez riche pour s'en passer,

Personne n'est assez pauvre pour ne pas le mériter.

Raoul FOLLEREAU

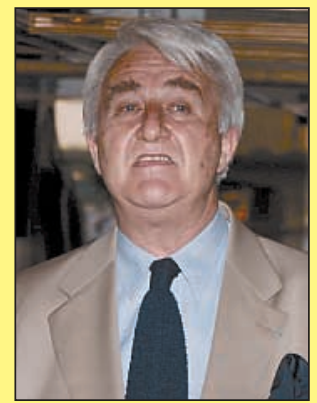

$\mathrm{U}$

n sourire met en jeu de nombreux composants du visage comme les lèvres, les dents, les gencives, les structures osseuses de la face, les muscles et même les yeux. La plupart de ces composants concerne directement nos professions. C'est pourquoi il a semblé indispensable à Alain BÉRY, à l'origine de ce projet, et à moi-même, qui y ai collaboré, de consacrer un numéro spécial à ce sujet.

Nous avons fait appel à des auteurs de grand talent représentant les différentes disciplines de notre profession mais aussi à des spécialistes comme Edwige ANTIER, pédiatre bien connue, pour aborder le problème du sourire chez l'enfant, de son évolution et de sa signification. De même, Marc Gérald CHOUKROUN a apporté sa contribution à l'éternel débat entre esthétique et cosmétique.

Pour respecter la tradition des AOS, odontologistes et stomatologistes se sont complétés dans le traitement des thèmes qui leur avaient été confiés. C'est ainsi que G. CLEDES, R. FELIZARDO et P. CARPENTIER ont décrit l'anatomie musculaire du sourire; Elisabeth DURSUN, Aurélie BESLOT et Marguerite-Marie LANDRU ont proposé la prévention et la thérapeutique chez le jeune patient ; Olivier HUE s'est chargé de l'aspect prothétique du problème ; Marc BERT ne pouvait qu'aborder les difficultés liées à l'implantologie et Julia COHEN-LÉVY, associée à Robert GARCIA, les traitements d'orthopédie dento-faciale.

De leur côté, les stomatologistes se sont chargés des aspects qui les concernent plus particulièrement. Thierry PIRAL a tenté de résoudre le délicat problème du sourire gingival et quant à Rémi LOCKHART et Jacques DICHAMP, ils envisagent les solutions chirurgicales apportées par les ostéotomies maxillaires.

Nous vous soumettons donc une approche qui se veut à la fois complète et originale des nombreux procédés et techniques qui peuvent être mis en œuvre pour protéger, corriger ou rétablir le sourire afin de faire bénéficier à nos patients de ce merveilleux outil de communication et de bien être qu'il représente. 
Et si toutefois, vous rencontrez quelqu'un qui ne sait plus sourire, Soyez généreux, donnez-lui le vôtre, Car nul n'a autant besoin d'un sourire que celui qui ne peut en donner aux autres.

Raoul FOLLEREAU 


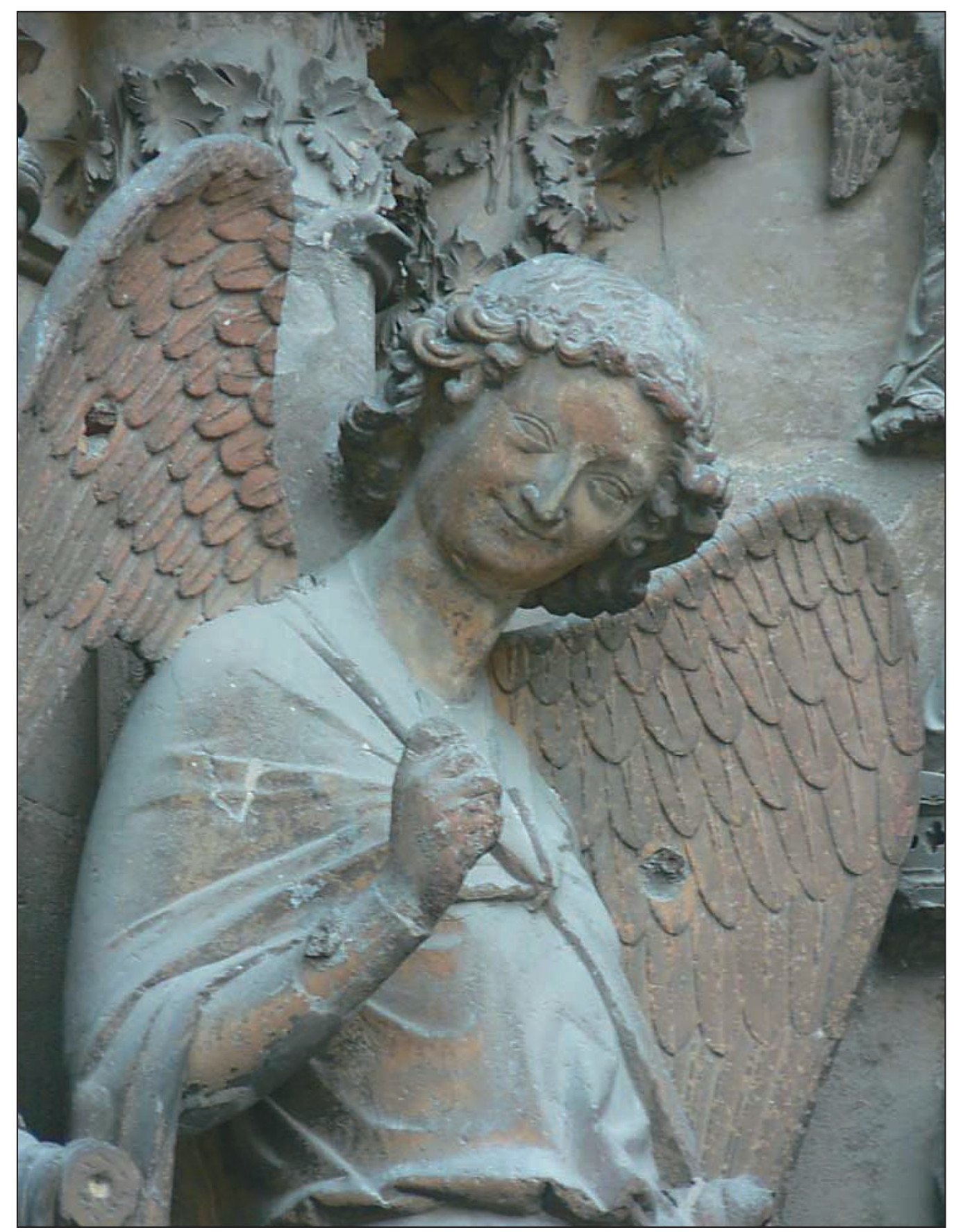

Le fameux «ange au sourire» de Reims, sur le portail Nord de la façade, endommagé par un obus pendant la $1^{\text {re }}$ guerre mondiale. 


\section{JUIN 2008}

93 PRÉAMBULE

H. OUVRARD

SPÉCIAL SOURIRE

le sourire de l'enfant :

son premier capital

E. ANTIER

SPÉCIAL SOURIRE

esthétique ou cosmétique?

M. G. CHOUKROUN

aesthetics or cosmetic?

111 SPÉCIAL SOURIRE

anatomie musculaire du sourire

G. CLÈDES, R. FELIZARDO, P. CARPENTIER

muscular anatomy of smile

SPÉCIAL SOURIRE

donner le sourire à nos jeunes patients : stratégies préventives et thérapeutiques

E. DURSUN, A. BESLOT, M.-M. LANDRU

to give the smile to our young patients:

preventive and therapeutic strategies

129 SPÉCIAL SOURIRE

le sourire en prothèse ou l'éloge du sourire

O. HUE

the smile is prosthodontics or the smile praise 


\section{SOMMAIRE}

143 SPÉCIAL SOURIRE

le sourire et les implants

M. BERT

smile and implants

155 SPÉCIAL SOURIRE

orthopédie dento-faciale

et architecture du sourire

J. COHEN-LÉVY, R. GARCIA

orthodontics, dento-facial orthopaedics and smile architecture

167 SPÉCIAL SOURIRE

le sourire gingival

T. PIRAL

the gummy smile

179 SPÉCIAL SOURIRE

la chirurgie du sourire : intérêt des ostéotomies maxillaires totales (Lefort I)

et segmentaires antérieures

R. LOCKHART, J. DICHAMP

the smile surgery: interest of total (Lefort I)

and anterior segmental maxillary osteotomies

193 LA VIE DE L'ASSOCIATION

programme scientifique 2008

jeudi 11 décembre 2008 :

les complications en implantologie 